\title{
Tocilizumab Treatment for Hospitalized Patients with Severe Covid-19 Pneumonia: A Retrospective Case-Control Study
}

\author{
Mohamed Aon ${ }^{1,2}$, Azeez Alzafiri ${ }^{1}$, Abdullah Alsaeedi ${ }^{1,3 *}$, Sherif Taha ${ }^{1,2}$, Abdelrahman Al Shammari ${ }^{1}$, Omar Al \\ Shammari $^{1}$, Mahmoud Tawakul ${ }^{1}$, Farah Al Shammari ${ }^{1}$, Najlaa Saeed ${ }^{1}$, Alaa Alenezi ${ }^{1}$, Manar Al Daas ${ }^{1}$, Thunayan \\ Al Dalmani ${ }^{1}$, Jarrah Al Shammari ${ }^{1}$, Adnan Alsarraf ${ }^{1}$, Eisa Aldhafeeri ${ }^{1}$, Mohamed M Ibrahim ${ }^{3}$, Humood Al \\ Shammari ${ }^{3}$, Omar Aldhafeeri ${ }^{4}$ and Ahmed Aoun ${ }^{5}$
}

${ }^{1}$ Department of Medicine, Jahra Hospital, Kuwait

${ }^{2}$ Department of Medicine, Cairo University, Egypt

${ }^{3}$ Department of clinical pharmacology, Jahra Hospital, Kuwait

${ }^{4}$ Medical student, NUI Galway, Ireland

${ }^{5}$ Department of Pediatrics, Cairo University, Egypt

Submission: February 19, 2021; Published: March 03, 2021

*Corresponding author: Dr. Abdullah Alsaeedi MD, FRCPC, FACP, FCCP, Pulmonary \& Sleep Medicine, PO Box 40206, Department of Medicine, Aljahra hospital 00004, Kuwait

Abstract

Background: Tocilizumab, through the blocking of interleukin-6 receptors, is hypothesized to be effective in the treatment of severe coronavirus disease 2019 (COVID-19). However, data on tocilizumab use in this setting is conflicting.

Objective: To evaluate the effect of tocilizumab treatment on the outcomes of patients with severe COVID-19 pneumonia.

Methods: A retrospective case-control study including 168 patients hospitalized with severe COVID-19 pneumonia at Al-Jahra hospital, Kuwait, from April 1st to June 30th. All patients received standard of care treatment. Patients in the tocilizumab group received tocilizumab infusion. The primary outcomes were death, need for mechanical ventilation and the proportion of patients with clinical improvement. The incidence of adverse events was monitored.

Results: Need for mechanical ventilation was higher in the tocilizumab group ( $48 \%$ vs. $24.3 \%, \mathrm{p}=0.002$ ). Clinical improvement was lower ( $54.1 \%$ vs. $71.4 \%)$ and number of deaths were higher $(29.6 \%$ vs. $10 \%)$ in the tocilizumab group ( $\mathrm{p}=0.009)$. There was no difference in the mean survival time between tocilizumab and control group (17.7 days vs. 19.2 days). There was no significant difference between the two groups regarding the incidence of infections $(6.1 \%$ vs. $2.9 \%, \mathrm{p}=0.471)$.

Conclusion: Our results suggest that use of tocilizumab in severe COVID-19 pneumonia does not provide clinical or mortality benefit. However, further research is needed to determine the ideal utilization of tocilizumab in the setting of severe COVID-19 disease.

Keywords: Tocilizumab; COVID-19; SARS-CoV-2; Pneumonia; Mechanical ventilation

Abbreviation: ALT: Alanine Aminotransferase; ARDS: Acute Respiratory Distress Syndrome; BCRSS: Brescia COVID-19 Respiratory Severity Scale; CI: Confidence Interval; COVID-19: Coronavirus Disease 2019; CRP: C-Reactive Protein; CSS: Cytokine Storm Syndrome; HFNC: High Flow Nasal Cannula; HR: Hazard Ratio; ICU: Intensive Care Unit, IL-6: Interleukin-6; IQR: Interquartile Range; LMWH: Low Molecular Weight Heparin; MV: Mechanical Ventilation; NIV: Non-Invasive Ventilation; PCR: Polymerase Chain Reaction; SARS-COV-2: Severe Acute Respiratory Syndrome Coronavirus-2; SD: Standard Deviation; SO2: Oxygen Saturation; SoC: Standard Of Care; SPSS: Statistical Package for the Social Sciences; TCZ: Tocilizumab

\section{Introduction}

Coronavirus disease 2019 (COVID-19) is a new disease caused by severe acute respiratory syndrome coronavirus 2 (SARS-CoV-2).
It started as a cluster of pneumonia cases in Wuhan, at the end of 2019, spreading to cause an epidemic throughout China, followed by an increasing number of cases all over the world creating 
a global pandemic [1]. The spectrum of COVID-19 symptoms is wide including asymptomatic, mild, severe, and critical disease. The proportion of severe infections is higher among hospitalized patients [2]. Severe disease is defined as infections associated with respiratory rate $\geq 30 / \mathrm{min}$, oxygen saturation $\leq 93 \%$ while breathing ambient air, and/or lung infiltrates $>50 \%$ on imaging. Critical disease is usually associated with respiratory failure, septic shock, and/or a syndrome of multiple organs dysfunction [3].

Among those with severe disease, one of the major complications and the leading causes of death is acute respiratory distress syndrome (ARDS) and the associated hypoxemic respiratory failure. In those who are critically ill, around half of cases will need invasive mechanical ventilation [4]. Another serious complication of severe COVID-19 is the cytokine storm syndrome (CSS) and the vigorous inflammatory response, characterized by elevated inflammatory markers (e.g., D-dimer, ferritin, and C-reactive protein [CRP]), and increased levels of proinflammatory cytokines including interleukin-6 (IL-6). A higher concentration of IL- 6 was found to be associated with higher in-hospital mortality [5,6]. IL-6 has a central role in acute inflammation. It stimulates widespread hematopoiesis, T-cell population expansion, activation and differentiation of B-cells, and induces the hepatic acute phase response with release of CRP, fibrinogen, and hepcidin [7]. IL-6 is a key component in the pathogenesis of the CSS. The cytokine storm and the elevated levels of IL- 6 damage the lungs and play a major role in the pathogenesis of ARDS and the multiple-organ failure syndrome in COVID-19 patients. Therefore, suppressing the IL-6 pathway is presumed to hinder the deterioration of patients and save patients' lives [8].

Tocilizumab (TCZ), a recombinant humanized IL-6 receptor monoclonal antibody, when administered to patients with severe COVID-19, was hypothesized to prevent the cytokine storm by blocking the IL-6 signal transduction pathway and reduce disease severity [9]. At the time of this manuscript preparation, data describing the use of TCZ in severe COVID-19 disease was limited to case series and observational studies. Some retrospective cohort studies found lower death and/or Intensive care unit (ICU) admissions [10] and shorter duration of vasopressor support [11] among patients treated with TCZ. Other observational studies found that TCZ reduced mortality in non-intubated patients [12] and in patients who needed invasive mechanical ventilation [13]. In a retrospective observational study (179 patients in the TCZ group vs. 365 patients in the control group), TCZ (intravenous or subcutaneous) was associated with a decreased risk of invasive mechanical ventilation and death (adjusted hazard ratio $0 \cdot 61$; $\mathrm{p}=0 \cdot 020$ ), but at the expense of higher risk of infections ( $13 \%$ vs. $4 \%, \mathrm{p}<0 \cdot 0001)[14]$.

However, other observational studies did not identify a clinical improvement or a mortality benefit with TCZ usage $[15,16]$. On the contrary, results from ongoing randomized trials, mostly have been reported as a press release, showed no benefit of TCZ in COVID-19 pneumonia. Preliminary results from the first randomized, double-blind, placebo-controlled phase III trial investigating use of TCZ in COVID-19 pneumonia were disappointing. TCZ did not result in improvement of clinical status or reduction in mortality [17]. In this study we aim to compare the outcomes of patients with severe COVID-19 pneumonia treated with TCZ in addition to standard of care (SoC) management, with those who received SoC management only.

\section{Methods}

\section{Patients and setting}

We retrospectively included patients with laboratoryconfirmed SARS-CoV-2 infection (defined as a positive polymerase chain reaction [PCR] from nasopharyngeal swab) who were hospitalized with severe COVID-19 pneumonia (defined as clinical and/or radiological evidence of pneumonia and Oxygen saturation (SO2) $\leq 93 \%$ while breathing ambient air) at Al-Jahra hospital, Kuwait, from April 1st to June 30th. Demographic, clinical, and laboratory data were retrieved from the patients' electronic records at baseline and reassessed on day 14 ( \pm 7 days). All patients received SoC treatments, as per the local COVID-19 management protocol, which included: 1) oxygen therapy to maintain SO2 $\geq 94 \%$, 2) corticosteroids, 3) anticoagulation prophylaxis, and 4) antibiotics (doses and details are included in Table 2). Other treatment options were available (e.g., lopinavir/ ritonavir, hydroxychloroquine, colchicine, and convalescent plasma treatment) but not considered as a SoC treatments and were prescribed on case-by-case basis according to the best available evidence.

In relation with the increasing literature data on the off-label use of TCZ to treat severe COVID-19 pneumonia, local treatment protocols were updated with escalation of treatment with TCZ $(8 \mathrm{mg} / \mathrm{kg}, 60 \mathrm{~min}$ intravenous infusion, max $800 \mathrm{mg}$ ) in patients who lacked clinical improvement with worsening in respiratory status over 24-48 hours and requiring $>5 \mathrm{~L} / \mathrm{min}$ to keep SO2 $\geq 94 \%$. A second administration within 24 hours was given if no clinical improvement was observed. Pregnant females, children, patients with contraindications or known allergy to TCZ, and patients with poor prognosis (unlikely to survive $>48$ hours) were excluded from the TCZ treatment protocol. Contraindications to TCZ included known active tuberculosis, known active hepatitis B and/or hepatitis C infection, known active diverticulitis, known inflammatory bowel disease, systemic bacterial or fungal co-infection, concomitant immunosuppressive therapies, transaminases $>5$ times above reference values, neutropenia $(<500 X 109 / \mathrm{L})$, and thrombocytopenia $(<50,000$ 109/L).

This retrospective study compared two groups of patients: the "TCZ group" which included all patients who received SoC treatment plus TCZ, and the "control group" which included age, 
sex, and severity matched patients who received SoC treatment without TCZ. The control group patients were potentially eligible to TCZ treatment but did not receive the drug either because they were admitted before TCZ treatment protocol was in effect or because of the drug unavailability due to the high local requirements that created an intermittent shortage. All patients' data were anonymous. The study was approved by the institutional ethical and research committee.

\section{Outcomes}

Patients' clinical status was assessed using a seven-category ordinal scale. The primary outcomes were death, need for invasive mechanical ventilation (MV) and the proportion of patients with clinical improvement in each group. Clinical improvement was defined as live discharge from the hospital or decrease of points $(\geq 2)$ on the seven-category scale at end of follow-up compared with baseline. The scale stages are 1) discharged home alive; 2) hospitalized and not requiring supplemental oxygen; 3) hospitalized and requiring low-flow oxygen $(\leq 5 \mathrm{~L} / \mathrm{min}) ; 4)$ hospitalized and requiring high-flow oxygen $(>5 \mathrm{~L} / \mathrm{min}$ by face mask or non-rebreather mask); 5) hospitalized and requiring high flow nasal cannula (HFNC) or non-invasive ventilation (NIV); 6) hospitalized and requiring invasive mechanical ventilation; and 7) death.

Patients with scales 2-3 were stratified as having low oxygen requirements, while patients with scales 4-6 were considered as having high oxygen requirements. Outcomes were assessed when the patient was discharged, at the end of follow- up period which is 21days, or when the patient died. The occurrence of adverse events was monitored with a focus on microbiologically confirmed infections.

\section{Statistical analysis}

The target sample size required for this cohort study was 150 patients to ensure a confidence level of $95 \%$ and a study power of $80 \%$, when the anticipated incidence of outcome in control and tocilizumab groups is set at $20 \%$ and $5 \%$ respectively. We collected 180 patient records. After data validation and removal of duplicate data $(\mathrm{n}=12)$, we ended up with 168 patient records. Validated data was tabulated, entered, and analyzed using Statistical Package for the Social Sciences (SPSS), version 21.0 (SPSS Inc., Chicago, IL, USA).

Numerical data were expressed as mean \pm standard deviation (SD) or median and interquartile range (IQR) as appropriate. Chisquare $\chi 2$ or Fisher Exact test was used to test for categorical variables. Independent Samples Student t-test was used for normally distributed numerical variables while Mann-Whitney test was used for analysis of non-parametric variables. KaplanMeier curve was used to perform survival analysis and hazard ratio (HR) was calculated using Cox regression after control of confounders. The threshold of significance in our study was defined as $p$ value $<0.05$.

\section{Results}

Table 1: Baseline demographics, comorbidities, clinical characteristics, laboratory, and radiological findings.

\begin{tabular}{|c|c|c|c|}
\hline & Tocilizumab Group $(n=98)$ & Control Group $(n=70)$ & $P$ value \\
\hline Age (years), Mean $( \pm$ SD) & $57.21( \pm 12.45)$ & $55.7( \pm 14.38)$ & 0.472 \\
\hline \multicolumn{4}{|l|}{ Sex, number (\%) } \\
\hline Male & $76(77.6)$ & $48(68.6)$ & 0.19 \\
\hline Female & $22(22.4)$ & $22(31.4)$ & \\
\hline \multicolumn{4}{|l|}{ Comorbidities, number (\%) } \\
\hline Hypertension & $48(49)$ & $38(54.3)$ & 0.498 \\
\hline Diabetes mellitus & $45(45.9)$ & $41(58.6)$ & 0.1 \\
\hline Respiratory disease & $10(10.2)$ & $15(21.4)$ & $0.04^{*}$ \\
\hline Cardiac disease & $20(20.4)$ & $18(25.7)$ & 0.41 \\
\hline $\begin{array}{l}\text { Immunosuppressed (transplantation and/or immuno- } \\
\text { suppressive drugs) }\end{array}$ & $3(3.1)$ & $6(8.6)$ & 0.166 \\
\hline
\end{tabular}




\begin{tabular}{|c|c|c|c|}
\hline Active cancer & $2(2)$ & $1(1.4)$ & 0.625 \\
\hline Chronic kidney disease (GFR $<60 \mathrm{ml} / \mathrm{min}$ ) & $9(9.2)$ & $16(22.9)$ & $0.014^{*}$ \\
\hline Smokers & $22(22.4)$ & $19(27.1)$ & 0.48 \\
\hline Days of symptoms prior to admission, mean $( \pm$ SD) & $5.8 \pm 3.67$ & $5.56 \pm 2.76$ & 0.64 \\
\hline \multicolumn{4}{|l|}{ Symptoms at baseline, number (\%) } \\
\hline Fever & $48(49)$ & $53(75.7)$ & $<0.001^{*}$ \\
\hline Cough & $73(74.5)$ & $43(61.4)$ & 0.07 \\
\hline Dyspnea & $76(77.6)$ & $50(71.4)$ & 0.36 \\
\hline Sore throat & $13(13.3)$ & $11(15.7)$ & 0.65 \\
\hline Headache & $60(61.2)$ & $42(60)$ & 0.87 \\
\hline Myalgia & $62(63.3)$ & $35(50)$ & 0.08 \\
\hline Nausea and/or vomiting & $25(25.5)$ & $15(21.4)$ & 0.54 \\
\hline Diarrhea & $50(51)$ & $36(51.4)$ & 0.95 \\
\hline Anosmia and/or loss of taste & $43(43.9)$ & $38(54.3)$ & 0.18 \\
\hline \multicolumn{4}{|l|}{ Vital signs at baseline, mean $( \pm S D)$} \\
\hline Oxygen saturation \% (breathing ambient air) & $87.6( \pm 7)$ & $88( \pm 5.8)$ & 0.67 \\
\hline Temperature $\left({ }^{\circ} \mathrm{C}\right)$ & $37.7( \pm 0.8)$ & $37.8( \pm 0.8)$ & 0.72 \\
\hline Heart rate (beat per minute) & $96( \pm 17)$ & $94( \pm 19)$ & 0.68 \\
\hline Respiratory rate (breath per minute) & $25( \pm 6)$ & $27( \pm 6)$ & 0.06 \\
\hline Systolic blood pressure (mmHg) & $130( \pm 19)$ & $129( \pm 19)$ & 0.7 \\
\hline Diastolic blood pressure (mmHg) & $75( \pm 11)$ & $74( \pm 11)$ & 0.65 \\
\hline Mean arterial pressure $(\mathrm{mmHg})$ & $93( \pm 11)$ & $92( \pm 12)$ & 0.62 \\
\hline \multicolumn{4}{|l|}{ Laboratory results at baseline, median (IQR) } \\
\hline White cell count (NR: 4-10 X109/L) & $6.9(5.4-9.5)$ & $8(5.8-11.5)$ & 0.15 \\
\hline Neutrophils (NR: 2-7 X109/L) & $5.3(4-7.7)$ & $5.8(3.8-9)$ & 0.64 \\
\hline Lymphocytes (NR: 1-3 X109/L) & $0.9(0.6-1.2)$ & $0.9(0.7-1.7)$ & $0.04^{*}$ \\
\hline Platelets (NR: $150-410 \times 10^{9} / \mathrm{L}$ ) & $209(168-255)$ & 247 (183-330) & $0.03^{*}$ \\
\hline C-reactive protein (NR: $0-0.8 \mathrm{mg} / \mathrm{dl}$ ) & $9.7(5.3-16.2)$ & $10(5.1-14.7)$ & 0.78 \\
\hline Procalcitonin (NR: $<0.15 \mathrm{ng} / \mathrm{ml}$ ) & $0.2(0.05-0.5)$ & $0.18(0.07-0.5)$ & 0.92 \\
\hline Creatinine (NR: 44-120umol/L) & $81(67-103)$ & $76.8(66-101)$ & 0.45 \\
\hline
\end{tabular}


International Journal of Pulmonary \& Respiratory Sciences

\begin{tabular}{|c|c|c|c|}
\hline Uric acid (NR: 150-400umol/L) & $275.5(207-365.3)$ & $259(186-363.8)$ & 0.51 \\
\hline ALT (NR: 10-60 IU/L) & $36(27-68)$ & $37(21.5-60.5)$ & 0.42 \\
\hline AST (NR: 10-42 IU/L) & $47.5(34.5-75.3)$ & $41(25-70.5)$ & 0.07 \\
\hline Lactate dehydrogenase (NR: 95-200 IU/L) & $404.5(307-582.5)$ & $330.5(251.5-432.3)$ & $0.002 *$ \\
\hline Creatine kinase (NR: 22-269 IU/L) & $169.5(75.8-364)$ & $82(32-245.8)$ & $0.002^{*}$ \\
\hline Ferritin (NR: 14-310ng/ml) & $619.7(350.5-935)$ & $563.7(248.15-1020)$ & 0.73 \\
\hline D-dimers (NR: 0-231ng/ml) & $457.5(309.3-678.8)$ & $450(295.3-735.8)$ & 0.72 \\
\hline \multicolumn{3}{|l|}{ Chest X-ray abnormality, number (\%) } & \\
\hline Normal & $1(1)$ & $2(3)$ & 0.57 \\
\hline Abnormal (bilateral infiltrates and/or consolidation) & $97(99)$ & $68(97)$ & \\
\hline
\end{tabular}

* $\mathrm{P}$ value significant $<0.05 ; \mathrm{SD}$, standard deviation; GFR, glomerular filtration rate; IQR, interquartile range; NR, normal range; ALT, Alanine aminotransferase; AST, Aspartate Transaminase.

A total of 168 patients were included in this retrospective analysis, 98 patients in the TCZ group and 70 patients in the control group. Mean age in the TCZ group was 57.21 years (SD \pm 12.45 ) and in the control group was 55.7 years (SD \pm 14.38 ). Age and sex distribution were similar between the two groups as shown in Table 1 . The mean duration between the onset of symptoms and hospitalization was 5.8 days (SD \pm 3.67 ) in the TCZ group and 5.56 days ( $\mathrm{SD} \pm 2.76$ ) in the control group. The mean oxygen saturation while breathing ambient air was $87.6 \%(\mathrm{SD} \pm 7$ ) in the TCZ group and $88 \%$ (SD \pm 5.8 ) in the control group. (Table 1) shows variables that describe the make-up of each group at baseline.

In general, the distribution of variables across groups was balanced, including comorbidities, smoking status, symptoms, vital signs, oxygen saturation, laboratory, and radiological findings. However, few differences were noticed between the two groups. Respiratory disease and chronic kidney disease were more common among the controls ( $\mathrm{p}=0.04$ and 0.014 , respectively). Markers of inflammation (e.g., CRP, D-dimer, and ferritin) were high in both groups with no statistically significant difference between the two groups. However, the TCZ group patients tended to have lower lymphocyte count $(\mathrm{p}=0.04)$, lower platelet count $(\mathrm{p}=0.03)$, and higher levels of lactate dehydrogenase and creatine kinase ( $p=0.002$ and 0.002 , respectively) (Table 1 ).

All patients received background $\mathrm{SoC}$ treatments during hospitalization as detailed in Table 2. All patients in both groups received low molecular weight heparin (LMWH). Almost $90 \%$ of the patients received steroids in both groups. Antibiotics were used more frequently in the control group ( $97 \%$ vs. $80.6 \%$, $\mathrm{p}=0.001$ ). Although no major differences were observed in terms of clinical features and oxygen saturation between the two groups at baseline, the need for high flow oxygen therapy ( $>5 \mathrm{~L} / \mathrm{min}$ ) at baseline was significantly higher in the TCZ group $(82.7 \%$ vs. $54.3 \%, \mathrm{p}<0.0001$ ).

Non-standard treatments included: colchicine which was prescribed in a majority of patients in both groups (TCZ group $83.7 \%$ vs. control group $74.3 \%, \mathrm{p}=0.135$ ), convalescent plasma treatment which was used more commonly in the control group ( $22.9 \%$ vs. $10.2 \%, \mathrm{p}=0.025)$, and other treatments as shown in (Table 2). Among the patients who received TCZ, the most frequently prescribed dosage was $600 \mathrm{mg}$ (69 patients, $70.4 \%$ ) and nine patients (9.2\%) required a second dose of TCZ.

(Table 3) shows the outcomes at the end of the followup period. Need for mechanical ventilation was higher in the TCZ group ( $48 \%$ vs. $24.3 \%, \mathrm{p}=0.002$ ). The median duration of mechanical ventilation was higher among patients in the TCZ group (10 days vs. 8 days, $\mathrm{p}=0.028)$. Fifty-three patients $(54.1 \%)$ achieved clinical improvement in the TCZ group versus 50 (71.4\%) in the control group and thirty-six patients died, 29 (29.6\%) in the TCZ group and 7 (10\%) in the control group. The difference between the two groups was statistically significant $(p=0.009)$. As compared to the control group, more patients treated with TCZ needed ICU admission ( $56.1 \%$ vs. $40 \%, \mathrm{p}=0.039$ ) and vasopressor support ( $48 \%$ vs. $24.3 \%$, $\mathrm{p}=0.002$ ). The median length of hospital stay was longer in patients receiving TCZ treatment (12.5 vs. 10 days, $\mathrm{p}=0.018$ ).

We observed an improvement in the level of CRP, but not in ferritin or D-dimer levels after TCZ treatment as shown in (Figure 1). However, this laboratory improvement was not associated with a clinical benefit. We identified 37 patients with laboratory 
criteria suggesting CSS, defined as any two out of the following three criteria: serum ferritin $>900 \mathrm{ng} / \mathrm{ml}$, CRP $>10 \mathrm{mg} / \mathrm{dl}$, and Ddimer level $>1500 \mathrm{ng} / \mathrm{ml}$ [18], 16/70 in the SoC group and 21/98 in the TCZ group. Among those patients, treatment with TCZ was Table 2: Treatments received during hospitalization. not associated with benefit regarding need for MV or clinical improvement $(57.1 \%$ vs. $37.5 \%, \mathrm{p}=0.236 ; 42.9 \%$ vs. $62.5 \%$, $\mathrm{p}=0.236$, respectively).

\begin{tabular}{|c|c|c|c|}
\hline & Tocilizumab Group $(n=98)$ & Control Group $(n=70)$ & $P$ value \\
\hline \multicolumn{4}{|l|}{ Standard of care treatments, number (\%) } \\
\hline Low oxygen requirements (scale 2-3) & $17(17.3)$ & $32(45.7)$ & $<0.001^{*}$ \\
\hline High oxygen requirements (scale 4-6) & $81(82.7)$ & $38(54.3)$ & \\
\hline Antibiotics $^{1}$ & $79(80.6)$ & $68(97)$ & $0.001^{*}$ \\
\hline Corticosteroids $^{2}$ & $88(89.8 \%)$ & $64(91.4 \%)$ & 0.72 \\
\hline $\mathrm{LMWH}^{3}$ & $98(100)$ & $70(100)$ & NA \\
\hline \multicolumn{4}{|l|}{ Non-standard treatments, number (\%) } \\
\hline Hydroxychloroquine $^{4}$ & $6(6.1)$ & $3(4.3)$ & 0.736 \\
\hline Lopinavir-ritonavir ${ }^{5}$ & $6(6.1)$ & $2(2.9)$ & 0.471 \\
\hline Colchicine $^{6}$ & $82(83.7)$ & $52(74.3)$ & 0.135 \\
\hline Convalescent plasma $^{7}$ & $10(10.2)$ & $16(22.9)$ & $0.025^{*}$ \\
\hline
\end{tabular}

${ }^{*} \mathrm{P}$ value significant $<0.05$; LMWH, low molecular weight heparin; NA, not applicable; ${ }^{1}$ if suspected bacterial infections, given for 5 days or until bacterial infection is excluded by negative cultures and/or normal procalcitonin levels, whichever comes first; ${ }^{2}$ dexamethasone 6 mg as a standard dose or $15 \mathrm{mg}$ if patient had evidence of moderate-to-severe acute respiratory distress syndrome $\left(\mathrm{PaO}_{2} / \mathrm{FiO}_{2} \leq 200 \mathrm{mmHg}\right)$, with equivalent dose of methylprednisolone used if dexamethasone was not available; ${ }^{3}$ standard prophylactic dose adjusted according to renal function (enoxaparin $40 \mathrm{mg}$ daily) or intermediate dose (usual daily dose twice daily) for patients with very high D-dimer levels (>1500ng/ml), extreme weight $>120 \mathrm{~kg}$, or patients who are critically ill; ${ }^{4}$ Dose is $400 \mathrm{mg}$ twice on day 1 , followed by $200 \mathrm{mg}$ twice per day on days $2-5 ;{ }^{5}$ Dose is $400 / 100 \mathrm{mg}$ twice per day for 7 days; ${ }^{6}$ Dose is $0.5 \mathrm{mg}$ twice per day for 7 days; ${ }^{7}$ Transfusion of 2 units, 12 hours apart.

Table 3: Outcomes at the end of the follow-up period.

\begin{tabular}{|c|c|c|c|}
\hline & Tocilizumab Group $(n=98)$ & Control Group $(n=70)$ & $P$ value \\
\hline \multicolumn{4}{|l|}{ Mechanical ventilation } \\
\hline Need for MV, number (\%) & $47(48)$ & $17(24.3)$ & $0.002 *$ \\
\hline Duration of $\mathrm{MV}^{1}$ (days), median (IQR) & $10(7-14)$ & $8(5.5-15.5)$ & $0.028^{*}$ \\
\hline \multicolumn{4}{|l|}{ Clinical outcomes, number (\%) } \\
\hline Clinical improvement $^{2}$ & $53(54.1)$ & $50(71.4)$ & \multirow{3}{*}{$0.009^{*}$} \\
\hline No clinical improvement & $16(16.3)$ & $13(18.6)$ & \\
\hline Hospital mortality & $29(29.6)$ & $7(10)$ & \\
\hline \multicolumn{4}{|l|}{ ICU admission } \\
\hline Need for ICU, number (\%) & $55(56.1)$ & $28(40)$ & $0.039 *$ \\
\hline Duration of ICU stay ${ }^{3}$ (days), median (IQR) & $12(8-15)$ & $8(6-16.75)$ & 0.227 \\
\hline Need for vasopressor support, number (\%) & $47(48)$ & $17(24.3)$ & $0.002^{*}$ \\
\hline Length of hospital stay (days), median (IQR) & $12.5(8.75-18)$ & $10(7.75-14)$ & $0.018^{*}$ \\
\hline
\end{tabular}

${ }^{*} \mathrm{P}$ value significant $<0.05$; MV, mechanical ventilation; IQR, interquartile range; ICU, intensive care unit; ${ }^{1}$ In those who needed MV; ${ }^{2}$ Live discharge from hospital or decrease of points $(\geq 2)$ on the seven-category scale at end of follow-up compared with baseline; ${ }^{3}$ In those who needed ICU admission. 


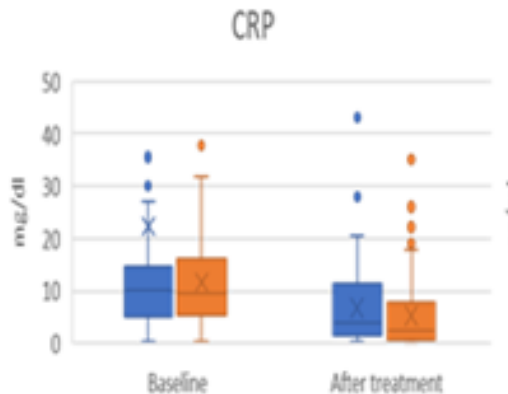

WOPSOC ICRPTC
Ferritin

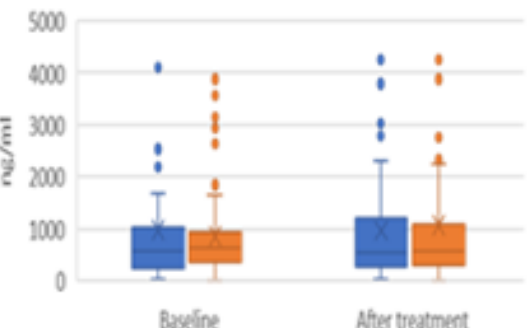

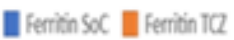

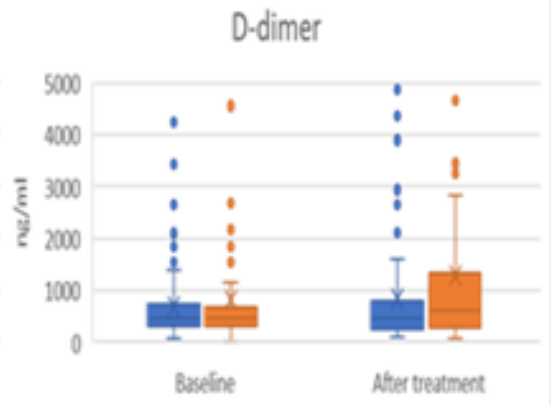

DD-iner SoC DD.dine TQ

\begin{abstract}
Parameter, median (IQR)
CRP, baseline

CRP, after treatment

Ferritin, baseline

Ferritin, after treatment

D-dimer, baseline

D-dimer, after treatment
\end{abstract}

TCZ group

$9.7(5.3-16.2)$
$2.3(0.7-7.7)$
$619.7(350.5-935)$
$584.3(294.5-1079.6)$
$457.5(309.3-678.8)$
$606(279.3-1328.3)$

SoC group

$10(5.1-14.7)$
$3.9(1.6-11.4)$
$563.7(248.15-1020)$
$540(265-1196)$
$450(295.3-735.8)$
$457.5(251.5-793)$

$P$ value

0.78

$0.019 *$

0.73

0.57

0.72

0.07

Figure 1: Changes in the values of CRP, ferritin, and D-dimer at baseline and after treatment in SoC and TCZ groups. Follow-up of laboratory parameters was done at discharge, death, or at the end of follow- up period which is 21 days. *Significant $<0.05$; IQR, interquartile range; CRP, C-reactive protein; TCZ, tocilizumab; SoC, standard of care.

Kaplan-Meier survival curves are presented in (Figure interval [CI] 0.94-6.35; p=0.06), after adjustment for age, sex, 2). There was no difference in the mean survival time after hospitalization between the 2 groups (TCZ group 17.7 days vs. SoC group 19.2 days). Hazard ratio of death [HR]: 2.5, 95\% confidence oxygen requirements at baseline, and CSS laboratory criteria by Cox regression.

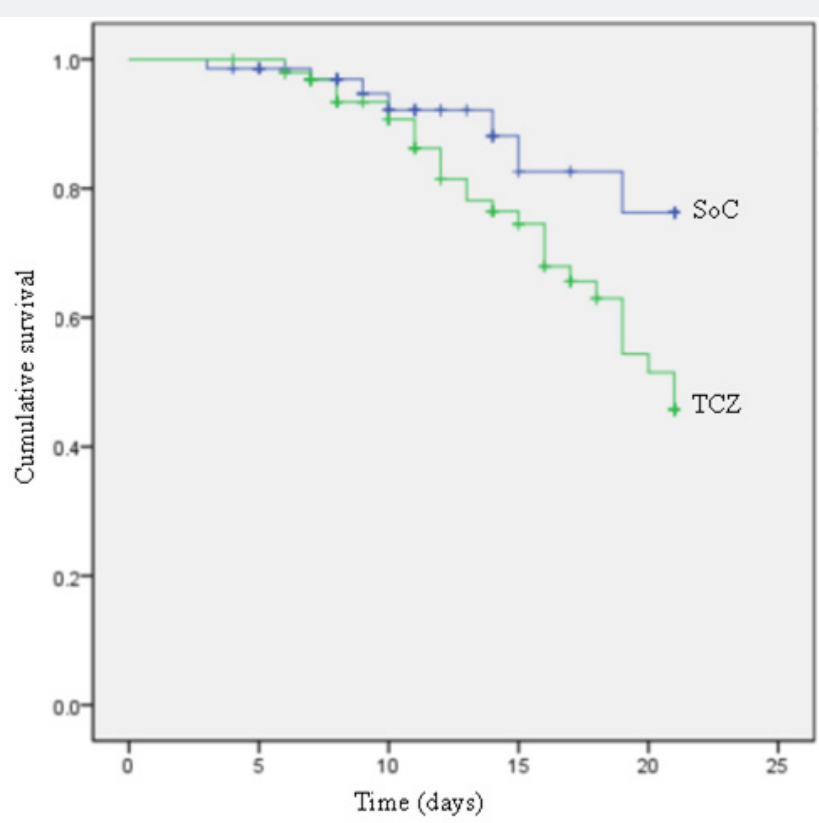

Figure 2: Survival curves for tocilizumab (TCZ) vs. standard of care (SoC) groups. 
Few unfavorable effects were observed following tocilizumab treatment. No tocilizumab infusion reactions were observed. Among patients treated with TCZ, 6 patients (6.1\%) developed microbiologically confirmed bacterial infections compared to 2 patients $(2.9 \%)$ in the SoC group, and the difference was not statistically significant $(\mathrm{p}=0.471)$. Sites of infections are shown in (Figure 3). Follow-up of Alanine aminotransferase (ALT) levels were higher among patients treated with TCZ as shown in (Figure 3).

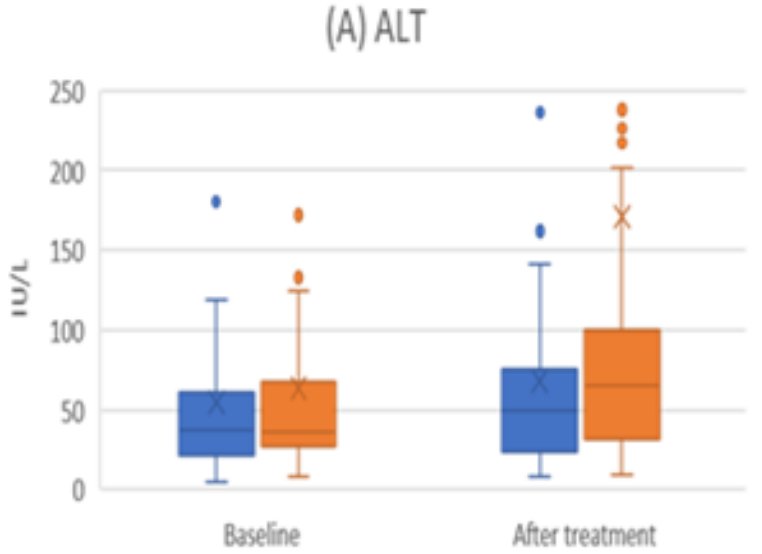

ALISOC ALITCZ

\section{(B) Microbiologically confirmed infections}

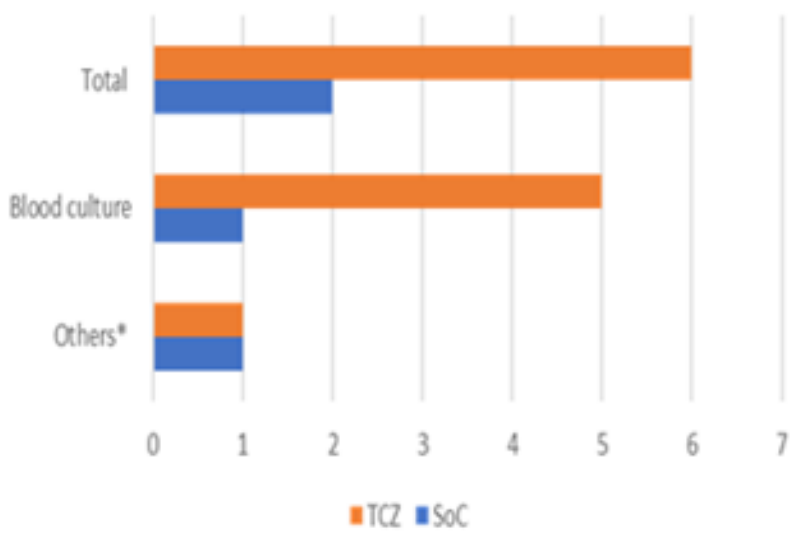

SoC group

$37(21.5-60.5)$

$49.5(24-75.3)$

\section{P value}

0.42

$0.009 * * *$
$36(27-68)$

$65(31.5-100.3)$

Figure 3: Adverse events observed with TCZ vs. SoC group. (A) level of ALT, (B) Sites of infections. *Tracheal secretion culture in the SoC group and wound swab culture in the TCZ group; ** Significant $<0.05 ; A L T$, alanine aminotransferase; IQR, interquartile range; TCZ, tocilizumab; SoC, standard of care.

\section{Discussion}

In the absence of a clear evidence-based recommendation, anti-inflammatory treatments (e.g., tocilizumab) are frequently offered to patients with severe COVID-19 pneumonia aiming not to overwhelm MV and ICU resources and to improve survival. It can be ethically appropriate to offer patients an investigational intervention outside clinical trials, provided that no established effective treatment exists, the use of the intervention is monitored, and the outcomes are recorded and shared [19]. This study describes our real-world experience with the use of tocilizumab in severe COVID-19 pneumonia.

We examined the clinical characteristics and outcomes of 98 patients with severe COVID-19 pneumonia who received TCZ added to SoC treatment compared to 70 patients with severe COVID-19 pneumonia receiving SoC alone. The TCZ treatment protocol we used to consist of a dose of $8 \mathrm{mg} / \mathrm{kg}$ (max $800 \mathrm{mg}$ ), given as a $60 \mathrm{~min}$ intravenous infusion. A similar weight-based treatment protocol was used in many studies of COVID-19 patients $[16,18,20-22]$.
Different weight-based dosing was used in other studies e.g. $4-8 \mathrm{mg} / \mathrm{kg}$ (max 400mg) [11]. Some studies used the subcutaneous formulation in a dose of $324 \mathrm{mg}$ if the intravenous formulation was not available $[14,23,24]$, while other studies used a low dose of $400 \mathrm{mg}$ regardless of the body weight $[15,23]$ or if the body weight was less than $60 \mathrm{~kg}$ [25]. The variability in dosing regimens makes it difficult to compare these studies and to assess the impact of this treatment.

All retrospective studies on TCZ used cohorts of hypoxic patients with severe COVID-19 infection. COVID-19 patients can be categorized, based on their oxygen needs, into mild disease with no need for oxygen to maintain target saturation; moderate disease with needs up to $5 \mathrm{~L} / \mathrm{min}$; severe disease with needs up to $10 \mathrm{~L} / \mathrm{min}$; very severe disease if patient needs a non-rebreather mask or high-flow nasal cannula to maintain target saturation; and critical disease in patients on mechanical ventilation [12]. We used an ordinal scale to express these categories and we considered patients who need $>5 \mathrm{~L} / \mathrm{min}$ as having high oxygen requirements. In spite of our attempts to match the 2 groups at 
baseline regarding their age, sex, comorbidities, baseline oxygen saturation, laboratory, and radiological findings, the need for high flow oxygen therapy was higher in the TCZ group (82.7\% vs. $54.3 \%, \mathrm{p}<0.001$ ). This difference is due to the probable presence of unmeasured confounding variables.

Previous studies on TCZ tested populations of diverse categories of oxygen therapy at baseline. In a cohort of patients treated with TCZ, $30 \%$ of patients required invasive or noninvasive ventilation and 53\% required high flow oxygen therapy [16]. In another study, $93.7 \%$ of patients who received TCZ was on high flow oxygen therapy and $63 \%$ of patients needed mechanical ventilation [12]. In a large case series including 100 patients treated with TCZ, all of them had acute respiratory failure requiring ventilatory support (Brescia COVID-19 Respiratory Severity Scale [BCRSS] $\geq 3$ ), $57 \%$ treated with NIV, and $43 \%$ required invasive MV [21]. Another study including 28 patients who received TCZ reported a baseline invasive MV rate of $75 \%$ and around $80 \%$ of the TZC group patients were on high flow oxygen therapy [11]. On the other hand, some studies included only patients with severe COVID-19 pneumonia and respiratory failure, not needing invasive mechanical ventilation $[15,23]$.

The heterogeneity of baseline oxygen needs and MV status of TCZ studies is another reason of the difficulty encountered when comparing these studies and their impact on clinical outcomes. We used SoC treatment protocol including corticosteroids, anticoagulation prophylaxis, and antibiotics. Treatments lacking supporting evidence were considered as a non-standard intervention as illustrated in (Table 2). Retrospective studies comparing TCZ add to SoC vs. SoC alone used various protocols of SoC management. Hydroxychloroquine was used as background treatment in many studies, but in our study, it was not considered a part of SoC [11-15,21,22]. Unlike our study, other studies used lopinavir/ritonavir as part of SoC management [14,15,21,23]. Similar to our protocol, some studies used prophylactic dose of anticoagulation as SoC $[14,15,22]$, while others used full anticoagulation in around half of the study population [12].

We used 2 dosing regimens of LMWH, the prophylactic dose and the intermediate dose. Our local practice is in accord with the recommendation that all hospitalized patients with COVID-19 should get thromboprophylaxis with LMWH with dose adjustment for renal functions and body weight. For critically ill patients, intermediate-intensity dose strategy may be used (usual daily LMWH dose twice daily). This recommendation is based mainly on expert opinion. However, intermediate-intensity or a therapeutic-intensity dose strategy should be practiced as a part of ongoing epidemiologic studies or clinical trials [26].

Immunomodulatory agents are potentially effective for the treatment of severe COVID-19 infection associated with a cytokine storm or a vigorous inflammatory response. However, the combination of these agents is still not clearly recommended.
No randomized trials till now addressed this issue. Some studies used steroids with TCZ, but had different protocols e.g., tapering dose of methylprednisolone (mg/kg) [22], dexamethasone (20mg/ day) [21], or high- dose intravenous methylprednisolone (250mg on day 1 followed by $80 \mathrm{mg}$ on days 2-5) [18].

A non-randomized study comparing TCZ and combination therapy of steroids and TCZ found that TCZ was associated with lower hazard for the primary outcome (death and need for MV), while combination therapy was not associated with a significant hazard difference. There was no difference seen in the rates of gastrointestinal bleeding or bacterial infections [27]. Another observational study found that adjunctive treatment with tocilizumab, steroids, or both added to SoC can improve outcomes in non-intubated patients with COVID-19 pneumonia. However, the rate of microbiologically documented infections was higher among patients who received TCZ/steroids when compared to SoC treatment alone [28]. We used a dose of $6 \mathrm{mg}$ dexamethasone as a standard and a higher dose for patients with evidence of moderate-to-severe ARDS (PaO2/FiO2 $\leq 200 \mathrm{mmHg}$ ) [29].

It is now recommended to use dexamethasone $6 \mathrm{mg}$ daily for 10 days or until discharge for all COVID-19 patients who are on supplemental oxygen or ventilatory support [30]. The variable treatment regimens used with TCZ make it difficult to assess the benefits of TCZ based on the results of these studies. Further data are needed for tocilizumab in patients receiving corticosteroids. Randomized studies are necessary to establish the safest treatment options.

Our study revealed no clinical improvement or mortality benefit in patients who received tocilizumab in the setting of severe COVID-19 pneumonia. Although the observational study design cannot prove causation, the higher oxygen needs at baseline in the TCZ group could partially contribute to the worse outcome. Most observational studies' data support the notion that TCZ use is associated with a benefit in severe COVID-19 infections. For example, a large case series found improvement of inflammatory parameters, clinical and respiratory condition at 10 days after TCZ treatment [21]. One study found that combined death and/or ICU admission was lower with TCZ treatment ( $25 \%$ vs. $72 \% \mathrm{P}=0.002)$, with less need for MV (0\% vs. 32\%, P=0.006) [10]. Mortality benefit only for non-intubated patients was reported in one study ( $6 \%$ vs. $27 \%, P=0.024$ ) [12]. While others reported mortality benefit only for intubated patients $(45 \%$ reduction in hazard of death) [13]. Shorter duration of vasopressor support with TCZ treatment was described in another observational study [11].

These reports are not comparable largely as a consequence of the various tocilizumab dosage used, the different baseline characteristics of the population studied, and the different concomitant medications. In our study, none of these outcomes were noticed, apart from improvement of CRP after TCZ treatment. 
A meta-analysis of observational studies including 1358 patients found a $12 \%$ lower mortality for COVID-19 patients treated with TCZ compared to patients who were not treated with TCZ [31]. Another analysis of a multicenter cohort study including 3924 ICU critically ill COVID-19 patients found that treatment with tocilizumab in the first 2 days of ICU admission reduces hospital mortality (HR, 0.71; 95\% confidence interval [CI], 0.56-0.92) [32].

Quite the opposite, earliest results from randomized trials do not show a clear clinical benefit of TCZ. Preliminary results from the COVACTA trial were disappointing as TCZ did not result in improvement of clinical status or reduction in mortality [17]. Another randomized trial showed no effect of TCZ on preventing intubation or death (HR, 0.83 ; 95\% CI, 0.38 to 1.81 ; $\mathrm{P}=0.64$ ) [33]. In another open-label randomized trial that included non-ICU COVID-19 patients, TCZ did not reduce 28-day mortality compared with usual care. (adjusted HR, 0.92; 95\% CI 0.33-2.53) [34].

The discrepancy between observational and randomized trials underscores the complexities in interpreting observational data because of the effect of unmeasured confounders. Of interest was the observation that in spite of improvement in CRP levels after TCZ treatment, this was not associated with a clinical benefit. In our study, subgroup group analysis showed no benefit of TCZ in patients with laboratory evidence of CSS, perhaps suggesting that the treatment of disease severity based on different criteria, rather than CSS, may play a role in survival. No major adverse events were observed after TCZ treatment in our study. The higher ALT level after TCZ treatment was reported in other studies $[20,25]$ The observed higher level of ALT after TCZ treatment cannot be merely attributed to TCZ. The role of concomitant medications and the nature of the COVID-19 infection itself cannot be ignored.

The main concern about tocilizumab treatment is the occurrence of severe infections. In our study, there was no significant difference between the two groups regarding rates of infection. While some observational studies showed higher rates of infection associated with TCZ treatment $[13,14]$, other observational studies did not [15]. Early data from randomized trials did not reveal higher rates of infection [33,34]. Long-term studies of TCZ in patients with rheumatoid arthritis showed that TCZ has a good safety profile [35].

Our study has limitations including the retrospective nature of the study, the short follow-up duration, and the presence of concomitant therapies. These considerations preclude a clear assumption on the efficacy of tocilizumab use in severe COVID-19 patients. Strengths include the presence of a control group and the homogeneity of the baseline characteristics of the population studied. Finally, our findings suggest that use of tocilizumab in severe COVID-19 pneumonia does not provide a clinical benefit. However, we need to highlight that some factors may be confounding in the analysis of the direct effect of TCZ, thus our results should be considered with caution.

\section{Conclusion}

In our study, we did not detect improvement in the clinical status or survival benefit in patients receiving tocilizumab compared to standard management. Rate of infectious adverse events requires vigilant monitoring to assess long-term hazards. The results of ongoing randomized trials are awaited to establish the role of TCZ in the treatment of severe COVID-19 pneumonia. Research is needed to determine the ideal timing, the optimal dose and route of administration for TCZ. Other immunomodulators, including glucocorticoids, combined with TCZ should be assessed for additional benefit in larger studies.

\section{References}

1. Wang C, Horby PW, Hayden FG, Gao GF (2020) A novel coronavirus outbreak of global health concern. Lancet 395(10223): 470-473.

2. Richardson S, Hirsch JS, Narasimhan M, Crawford JM, Mc Ginn T, et al. (2020) Presenting Characteristics, Comorbidities, and Outcomes Among 5700 Patients Hospitalized With COVID-19 in the New York City Area. JAMA 323(20): 2052-2059.

3. Wu Z, Mc Googan JM (2020) Characteristics of and Important Lessons from the Coronavirus Disease 2019 (COVID-19) Outbreak in China: Summary of a Report of 72314 Cases from the Chinese Center for Disease Control and Prevention. JAMA 323(13): 1239-1242.

4. Wang D, Hu B, Hu C, Zhu F, Liu X, et al. (2020) Clinical Characteristics of 138 Hospitalized Patients With 2019 Novel Coronavirus-Infected Pneumonia in Wuhan, China. JAMA 323(11): 1061-1069.

5. Mehta P, Mc Auley DF, Brown M, Sanchez E, Tattersall RS, et al. (2020) COVID-19: consider cytokine storm syndromes and immunosuppression. Lancet 395(10229): 1033-1034.

6. Cummings MJ, Baldwin MR, Abrams D, Jacobson SD, Meyer BJ, et al. (2020) Epidemiology, clinical course, and outcomes of critically ill adults with COVID-19 in New York City: a prospective cohort study. Lancet 395(10239): 1763-1770.

7. Hunter CA, Jones SA, (2015) IL- 6 as a keystone cytokine in health and disease. Nat immunol 16(5): 448-457.

8. Ye Q, Wang B, Mao J (2020) The pathogenesis and treatment of the Cytokine Storm in COVID-19. J Infect 80(6): 607-613

9. Zhang C, Wu Z, Li JW, Zhao H, Wang GQ (2020) Cytokine release syndrome in severe COVID-19: interleukin-6 receptor antagonist tocilizumab may be the key to reduce mortality. Int J Antimicrob Agents 55(5): 105954.

10. Klopfenstein T, Zayet S, Lohse A, Balblanc JC, Badie J, et al. (2020) Tocilizumab therapy reduced intensive care unit admissions and/or mortality in COVID-19 patients. Med Mal Infect 50(5): 397-400.

11. Tariq Kewan, Fahrettin Covut, Mohammed J Al-J, Lori Rose, KV Gopalakrishna (2020) Tocilizumab for treatment of patients with severe COVID-19: A retrospective cohort study. E Clinical Medicine 24: 100418.

12. Rojas MG, Khalid M, Mukhtar O, Hashmi AT, Waheed MA, et al. (2020) Outcomes in patients with severe COVID-19 disease treated with tocilizumab: a case-controlled study. QJM 113(8): 546-550.

13. Somers EC, Eschenauer GA, Troost JP, Golob JL, Gandhi TN, et al. (2020) Tocilizumab for treatment of mechanically ventilated patients with COVID-19. Clin Infect Dis P: ciaa954. 
14. Guaraldi G, Meschiari M, Cozzi Lepri A, Milic J, Tonelli R, et. al (2020) Tocilizumab in patients with severe COVID-19: a retrospective cohort study. Lancet Rheumatol 2(8): e474-e484.

15. Campochiaro C, Della-Torre E, Cavalli G, De Luca G, Ripa M, et al. (2020) Efficacy and safety of tocilizumab in severe COVID-19 patients: a single-centre retrospective cohort study. Eur J Intern Med 76: 43-49.

16. Knorr JP, Colomy V, Mauriello CM, Ha S (2020) Tocilizumab in patients with severe COVID-19: A single-center observational analysis. J M Virol 92(11): 2813-2820.

17. Furlow Bryant (2020) COVACTA trial raises questions about tocilizumab's benefit in COVID-19. Lancet Rheumatol 2(10): e592.

18. Sofia R, Remy LMM, Cesar MC, Christel MPVD, Tom D, et al. (2020) Historically controlled comparison of glucocorticoids with or without tocilizumab versus supportive care only in patients with COVID-19associated cytokine storm syndrome: results of the CHIC study. Ann Rheum Dis 79(99): 1143-1151.

19. (2020) Off-label use of medicines for COVID-19. World Health Organization (WHO)

20. Price CC, Altice FL, Shyr Y, Koff A, Pischel L, et al., (2020) Tocilizumab Treatment for Cytokine Release Syndrome in Hospitalized Patients with Coronavirus Disease 2019: Survival and Clinical Outcomes. Chest 158(4): 1397-1408

21. Toniati P, Piva S, Cattalini M, Garrafa E, Regola F, et al. (2020) Tocilizumab for the treatment of severe COVID-19 pneumonia with hyperinflammatory syndrome and acute respiratory failure: A single center study of 100 patients in Brescia, Italy. Autoimmun Rev 19(7) 102568.

22. Colaneri M, Bogliolo L, Valsecchi P, Sacchi P, Zuccaro V, et al. (2020) Tocilizumab for Treatment of Severe COVID-19 Patients: Preliminary Results from SMAtteo COvid19 REgistry (SMACORE). Microorganisms 8(5): 695

23. Capra R, De Rossi N, Mattioli F, Romanelli G, Scarpazza C, et al. (2020) Impact of low dose tocilizumab on mortality rate in patients with COVID-19 related pneumonia. Eur J Internal Med 76: 31-35.

24. Sciascia S, Apra F, Baffa A, Baldovino S, Boaro D, et al. (2020) Pilot prospective open, single-arm multicentre study on off-label use of tocilizumab in patients with severe COVID-19. Clin Exp Rheumatol 38(3): 529-532.

25. Morena V, Milazzo L, Oreni L, Bestetti G, Fossali T, et al. (2020) Off- label use of tocilizumab for the treatment of SARS-CoV-2 pneumonia in Milan, Italy. Eur J Intern Med 76: 36-42.

26. Barnes GD, Burnett A, Allen A, Marilyn B, Nathan PC, et al. (2020) Thromboembolism and anticoagulant therapy during the COVID-19 pandemic: interim clinical guidance from the anticoagulation forum. J Thromb Thrombolysis 50(1): 72-81.

27. Jesus RB, Jeronimo P, Jordi C, Pablo R, Inmaculada J, et al. (2020) Treatment with tocilizumab or corticosteroids for COVID-19 patients with hyperinflammatory state: a multicentre cohort study (SAMCOVID-19). Clinical Microbiology and Infection 27(2): 244-252.

28. Mikulska M, Nicolini LA, Signori A, Di Biagio A, Sepulcri C, Russo C, et al (2020) Tocilizumab and steroid treatment in patients with COVID-19 pneumonia. PLoS One 15(8): e0237831.

29. Ferguson ND, Fan E, Camporota L, Antonelli M, Anzueto A, et al. (2012) The Berlin definition of ARDS: an expanded rationale, justification, and supplementary material. Intensive care Med 38(10): 1573-1582.

30. Horby, P, Lim WS, Emberson JR, Mafham M, Bell JL, et al. (2021) Dexamethasone in Hospitalized Patients with Covid-19 - Preliminary Report. The RECOVERY Collaborative Group. N Engl J Med 384(8): 693704.

31. Malgie J, Schoones JW, Pijls BG (2020) Decreased mortality in COVID-19 patients treated with Tocilizumab: a rapid systematic review and meta-analysis of observational studies. Clinical Infect Dis P: ciaa1445.

32. Gupta S, Wang W, Hayek SS, Chan L, Mathews KS, et al. (2020) Association Between Early Treatment with Tocilizumab and Mortality Among Critically Ill Patients With COVID-19. JAMA Internal Medicine 181(1): 41-51.

33. Stone JH, Frigault MJ, Serling Boyd NJ, Fernandes AD, Harvey L, et al (2020) Efficacy of Tocilizumab in Patients Hospitalized with Covid-19. N Engl J Med 383: 2333-2344.

34. Hermine O, Mariette X, Tharaux PL, Resche-Rigon M, Porcher R, et al (2021) Effect of Tocilizumab vs Usual Care in Adults Hospitalized With COVID-19 and Moderate or Severe Pneumonia: A Randomized Clinical Trial. JAMA Intern Med 181(1): 24-31.

35. Nishimoto N, Miyasaka N, Yamamoto K, Kawai S, Takeuchi T, et al. (2009) Long-term safety and efficacy of tocilizumab, an anti-IL-6 receptor monoclonal antibody, in monotherapy, in patients with rheumatoid arthritis (the STREAM study): evidence of safety and efficacy in a 5-year extension study. Ann Rheum Dis 68(10): 1580-1584.

This work is licensed under Creative

Commons Attribution 4.0 License

DOI: 10.19080/IJOPRS.2020.04.555646

\section{Your next submission with Juniper Publishers} will reach you the below assets

- Quality Editorial service

- Swift Peer Review

- Reprints availability

- E-prints Service

- Manuscript Podcast for convenient understanding

- Global attainment for your research

- Manuscript accessibility in different formats ( Pdf, E-pub, Full Text, Audio)

- Unceasing customer service

Track the below URL for one-step submission https://juniperpublishers.com/online-submission.php 